\title{
Imagining and naming rotated natural objects
}

\author{
JANICE E. MURRAY \\ University of Otago, Dunedin, New Zealand
}

\begin{abstract}
The present experiment examined whether subjects can form and store imagined objects in various orientations. Subjects in a training phase named line drawings of natural objects shown at six orientations, named objects shown upright, or imagined upright objects at six orientations. Time to imagine an upright object at another orientation increased the farther the designated orientation was from the upright, with faster image formation times at $180^{\circ}$ than at $120^{\circ}$. Similar systematic patterns of effects of orientation on identification time were found for rotated objects. During the test phase, all subjects named the previously experienced objects as well as new objects, at six orientations. The orientation effect for old objects seen previously in a variety of orientations was much reduced relative to the orientation effect for new objects. In contrast, substantial effects of orientation on naming time were observed for old objects for subjects who had previously seen the objects upright only or upright but imagined at different orientations. The results suggest that the attenuation of initially large effects of orientation with practice cannot be due to imagining and forming representations of objects at a number of orientations.
\end{abstract}

The time to name rotated line drawings of objects is highly dependent on the orientation of the object (e.g., Jolicoeur, 1985; Maki, 1986; McMullen \& Jolicoeur, 1992; Murray, 1995). For orientations between $0^{\circ}$ and $120^{\circ}$ of clockwise or counterclockwise rotation there is a linear increase in naming time the farther an object is rotated from the upright. This pattern of effects suggests that successful recognition requires a transformation of the input representation of a rotated object to the upright for comparison with orientation-dependent representations stored in memory. One candidate process for accomplishing this transformation is mental rotation, the analogue process assumed to underlie the orientation effect found for judgments of left-right reflection (Jolicoeur, 1985,1988 ). Inverted objects represent a deviation from the linear pattern described above; naming times for $180^{\circ}$ objects are faster than would be predicted by linear extrapolation from other orientations. This results in an Mshaped naming function and suggests the involvement of other orientation-dependent recognition processes for objects at $180^{\circ}$.

The dependence of naming time on orientation is most pronounced when the objects are viewed for the first time. As subjects become more familiar with a set of objects through repeated naming, the magnitude of the orientation effect diminishes significantly (e.g., Jolicoeur, 1985; Maki, 1986; McMullen \& Jolicoeur, 1992; Murray, Jolicoeur, McMullen, \& Ingleton, 1993). This practice or familiarity

This research was supported by a grant from the University of Otago Research Committee. I gratefully acknowledge the assistance of Paul Hanning and Ann Dowden with data collection. I thank Jeff Miller, Pierre Jolicoeur, Ruth Maki, and an anonymous reviewer for helpful comments. Correspondence regarding this paper may be sent to the author, Department of Psychology, University of Otago, Box 56, Dunedin, New Zealand (e-mail: jmur@rivendell.otago.ac.nz). effect is restricted to the objects in the experienced set and does not transfer to new objects (e.g., Jolicoeur, 1985), suggesting that something specific is learned about the objects that enables the viewer to minimize the effects of orientation in subsequent identifications.

One interesting feature of this practice effect which further suggests a highly flexible pattern-recognition system has been reported by Jolicoeur and Milliken (1989). They found that a reduced orientation effect on naming of rotated objects could be obtained for objects originally viewed upright. However, whether or not the practice effect is evidenced depends on the context in which the upright objects are viewed and encoded initially. Consider two different initial encoding contexts for upright objects. In one, all objects are named over blocks of trials in the context of upright objects only. In the second, the upright objects are named in the context of other objects that are rotated. When the upright objects are finally named at nonupright orientations, a reduced orientation effect is observed for the second context only. For the first encoding context, a robust orientation effect is obtained which does not differ from that shown for rotated objects named for the first time.

Jolicoeur and Milliken (1989) considered these results in terms of two possible accounts of the practice effect in general. One account suggests that although orientation-specific representations are used at the outset, subjects learn with practice which orientation-invariant attributes can serve as the basis for recognition of the specific items in the experienced set. If orientation-invariant representations do develop with familiarity with a set of objects, the results of Jolicoeur and Milliken suggest that in the case of upright objects this development occurs only in certain encoding contexts. A second account of practice effects is one in which the reduced orientation effect is the result of rotation to multiple representations 
of each familiar object. In this view, specific representations are encoded for each object at each orientation as it is experienced, and these orientation-specific representations can subsequently be used to identify the same objects. If an object has been seen at a number of orientations, that object at any new orientation will require less rotation to the nearest representation in memory for a successful match (see Tarr \& Pinker, 1989). This view would account for the finding that upright objects seen in an upright context do not show a reduced orientation when they are presented later for identification at nonupright orientations. How would such a view account for the finding of a reduced orientation effect when upright objects are viewed in the context of rotated objects? Jolicoeur and Milliken have suggested that subjects may adopt a strategy of rotating images of upright objects to a number of orientations and then storing representations of these rotated images in anticipation of seeing the objects at other orientations in later trials. It is reasonable to consider that subjects could adopt such a strategy given that the number of possible nonupright orientations was small (five) and that the orientation of the rotated objects and some of the upright objects changed from block to block.

The adequacy of this explanation rests on the assumption that subjects can indeed form and store imagined representations, and that they later make use of them in identification trials to reduce the effects of orientation. Several studies have shown that subjects can mentally rotate an image of an upright stimulus to a required orientation when given advance information about both the stimulus and the orientation, and that they can then use this rotated image to make a required reflection discrimination when immediately presented with the test stimulus (Bethell-Fox \& Shepard, 1988; Cooper, 1975; Cooper \& Shepard, 1973). However, nothing is known about whether these rotated images can be stored as longterm memory representations for later use in identification of the rotated stimuli. The main aim of this study was to assess this possibility. Over repeated blocks of training trials, subjects were asked to prepare rotated images of upright drawings of objects. During a later test phase, the same drawings were presented for naming at the orientations at which they had been imagined. If imagined multiple representations are formed during training and are subsequently used to identify the rotated objects, there should be a reduced effect of orientation on identification time for previously imagined objects relative to objects named for the first time.

\section{METHOD}

\section{Subjects}

Seventy-two students (43 females) of the University of Otago participated as subjects. All subjects had normal or corrected-to-normal vision and were native speakers of English. No subject had any previous experience with the stimuli. Testing occurred in a single session of approximately $50 \mathrm{~min}$.

\section{Stimuli and Apparatus}

The stimuli consisted of 96 line drawings of natural objects and animals with a distinct top and bottom. The drawings were selected from
Snodgrass and Vanderwart (1980) and were assigned randomly to two sets, A and B. Stored images of the drawings were created by scanning each drawing using a Hewlett-Packard Scanjet Plus. Each scanned drawing was then edited to produce uniform images for $0^{\circ}, 60^{\circ}, 120^{\circ}$, $180^{\circ}, 240^{\circ}$, and $300^{\circ}$ of clockwise rotation. The drawings were presented on a Zenith 1492 VGA color monitor as black line drawings on a light gray background. The drawings subtended an average $3^{\circ}$ of visual angle (range, $2.29^{\circ}-4.57^{\circ}$ ). Drawings of an additional six items were used during practice. Stimulus presentation and collection of responses was controlled by an NEC Powermate 386 computer with Micro Experimental Laboratory software (Schneider, 1988). Naming latency was detected by a voice-activated relay connected to a microphone.

\section{Procedure}

The experiment had two phases: training and test. In the training phase, 48 drawings were each presented once during a block of trials. This 48-trial block was presented five times to each subject. In the test phase, each subject received one block of 96 trials. Half the drawings in the test phase were those seen in the training phase (old objects); the remaining half were drawings not seen previously in the experiment (new objects). For half the subjects, Set A and Set B drawings served as old and new objects, respectively. For the other half, this assignment was reversed.

The subjects were divided into three groups-rotated, upright, and imagined-each of which received different training. The subjects in the rotated group named drawings shown at each of the six possible orientations from $0^{\circ}$ to $300^{\circ}$. There were equal numbers of drawings at each orientation, and each drawing was shown at only one orientation throughout the five blocks. Across subjects each drawing was presented equally often at each of the six orientations to control for any item-specific effects on orientation. Subjects in the upright group named drawings shown at $0^{\circ}$ only. For both the rotated and upright groups, a trial proceeded as follows. At the beginning of the trial, a small fixation cross was displayed in the center of the screen. Following a keypress by the subject, the screen was blanked for a $1.5-\mathrm{sec}$ interval, during which time the stimulus was written to the screen. Immediately following this interval, the screen was turned on at the top of the refresh cycle to reveal the drawing to the subject. The display remained in view until the subject responded. The subjects were instructed to name the drawing as quickly and accurately as possible. Naming latency was measured from the onset of the display screen to the onset of the subject's verbal response. Responses were detected by the voice-activated relay and recorded by the computer to the nearest millisecond. The trial concluded with the experimenter coding the response as correct, incorrect, or spoiled.

For the imagined group, a keypress following the fixation cross resulted in the display of a drawing of an upright object. The subjects were instructed to form an image of the object, and, when ready, to press the space bar in order to proceed. The drawing was then replaced with an orientation cue, which took the form of a black arrow presented in the center of the screen. This arrow indicated the orientation at which the previous drawing was to be imagined. The subject's instructions were to imagine the object at the designated orientation, with the arrow pointing to the top of the required rotated image. The subject was further instructed to press the space bar again when an image of the drawing at the required orientation was fully formed. It was stressed to the subjects that it was important that they form a clear rotated image of each drawing, because they would be tested on the images later. The orientation cue remained in view until the subject responded. The time to form the rotated image was measured from the onset of the orientation cue to the subject's keypress. There were equal numbers of objects imagined at each of the six possible orientations, and each drawing/orientation combination was matched to one in the rotated condition.

All subjects were treated identically in the test phase. The subjects received a sixth and final block of trials in which they were instructed to name rotated drawings as quickly and accurately as possible. Both old and new objects were presented in equal numbers at the six orientations, $0^{\circ}-300^{\circ}$. For the rotated and imagined groups, the old drawings were named at the orientation at which they had been named and imagined respectively in training. In the upright group, the old drawings were named at the same orientations, which were obviously different 
from those experienced in training. Both naming latency and accuracy were recorded as in the training phase.

Each subject was familiarized with the procedures and the use of the voice-activated relay in six practice trials. In addition, the subjects in the imagined group were initially given six trials demonstrating what rotated images should look like following presentation of the upright drawing and orientation cue.

\section{RESULTS}

Throughout this article, to analyze the linear increase in response time (RT) over orientation, the contrast weights of $-6,-1,4,4,-1$ were applied to the results for $0^{\circ}, 60^{\circ}, 120^{\circ}, 240^{\circ}$, and $300^{\circ}$, respectively, unless otherwise indicated. The data from $180^{\circ}$ were not included in the analyses of the linear effect of orientation on naming time, since the specific prediction of linearity is for $0^{\circ}$ to $120^{\circ}$ and then linearity from $240^{\circ}$ to $300^{\circ}$.

Naming latencies less than $300 \mathrm{msec}$ or greater than $3,000 \mathrm{msec}$, and image formation latencies less than $300 \mathrm{msec}$ or greater than $8,000 \mathrm{msec}$, were considered outliers. This resulted in a loss of less than $1 \%$ of the data. The percentage of outliers did not vary systematically with orientation. The mean RT for correct responses was calculated for each orientation within each training block, and for each orientation for both old and new drawings in the test phase.

The RT data from the test phase for each group, shown in Figure 1, were considered in three separate repeatedmeasures analyses of variance (ANOVAs) in which item type and orientation were factors. (Note that the results for $0^{\circ}$ and $360^{\circ}$ represent the same data and are presented to help judge the symmetry around $180^{\circ}$. The data for $0^{\circ}$ were considered only once in any analysis.) Corresponding analyses of error rates were also carried out. In all cases, none of the analyses suggested any speed-accuracy tradeoffs. The error rates for each group are reported below following each associated RT analysis.

The results for the rotated group were analyzed first. The overall effect of orientation was significant $[F(5,115)$ $\left.=4.31, M S_{\mathrm{e}}=10,676, p<.001\right]$ and had a strong linear component across departure from the upright $[F(1,23)=$ $\left.20.99, M S_{e}=9,553, p<.001\right]$. The time to name old drawings $(887 \mathrm{msec}$ ) was shorter than the time to name new drawings $(1,165 \mathrm{msec})\left[F(1,23)=163.02, M S_{\mathrm{e}}=\right.$ $33,965, p<.001]$. Most importantly, the magnitude of the orientation effect was larger for new drawings than for old drawings, as tested by both the item type $\times$ orientation interaction $\left[F(5,115)=2.46, M S_{\mathrm{e}}=14,459\right.$, $p<.036]$ and the item type $\times$ orientation $_{\text {linear }}$ interaction $\left[F(1,23)=5.71, M S_{\mathrm{e}}=15,026, p<.025\right]$. This expected result confirms the previous findings of a reduced orientation effect that is specific to the drawings experienced during training (Jolicoeur, 1985; Jolicoeur, 1988; Jolicoeur \& Milliken, 1989). The corresponding percent error rates for the six orientations were $3.5 \%, 1.0 \%, 3.5 \%$, $3.0 \%, 1.5 \%$, and $2.7 \%$ for old drawings, and for new, $7.5 \%, 5.9 \%, 6.2 \%, 8.6 \%, 4.6 \%$, and $5.9 \%$.

The results for the upright group also conform to expectancies based on previous findings (Jolicoeur \& Mil-
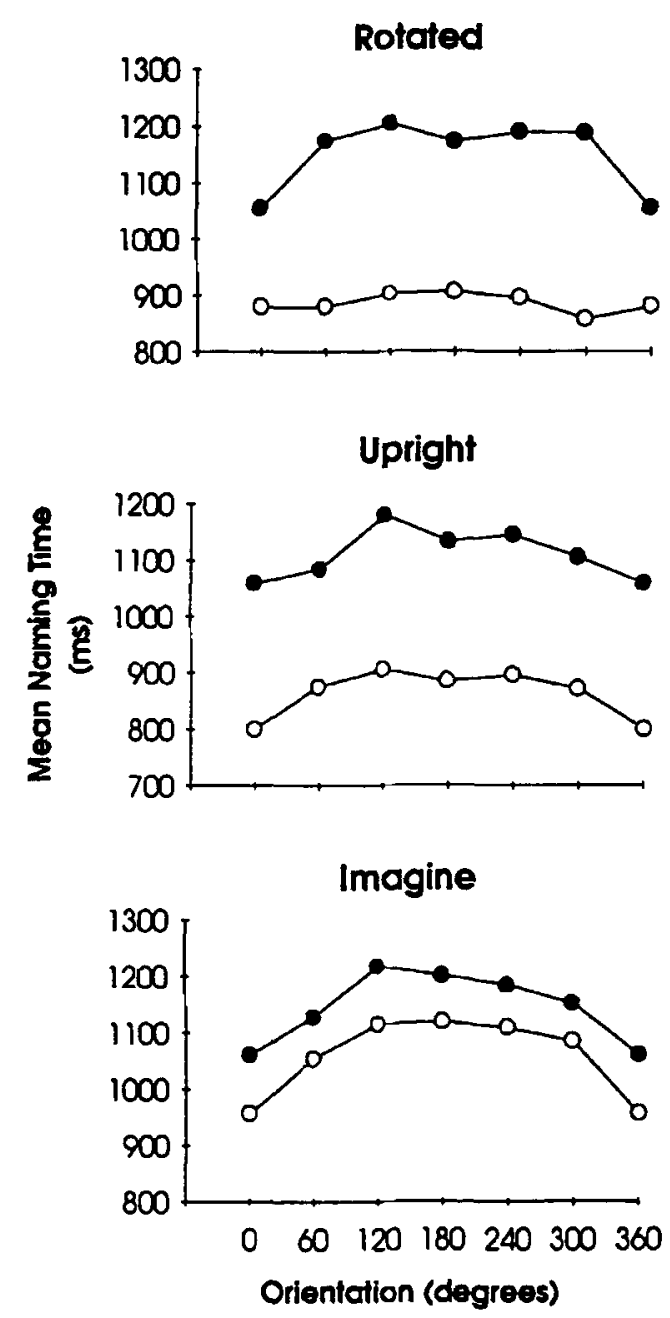

Figure 1. Mean naming time for old (open circles) and new (filled circles) objects at each orientation in the test phase for each group.

liken, 1989). The significant effect of orientation on naming time $\left[F(5,114)=5.92, M S_{\mathrm{e}}=12,480, p<.001\right]$, which had a strong linear component $[F(1,23)=41.25$, $\left.M S_{\mathrm{e}}=8,337, p<.001\right]$, did not differ across old and new drawings $(F<1)$, nor was there any difference in the slopes of the linear effect for the two types of drawings $(F<1)$. Finally, old drawings were named faster than new drawings (872 vs. $1,118 \mathrm{msec})[F(1,23)=367.94$, $\left.M S_{\mathrm{e}}=11,856, p<.001\right]$. The corresponding percent error rates for the six orientations were $2.0 \%, 5.8 \%, 3.5 \%, 2.1 \%$, $7.0 \%$, and $2.5 \%$ for old drawings, and for new, $4.6 \%$, $10.7 \%, 9.8 \%, 13.5 \%, 8.8 \%$, and $13.3 \%$.

The question of primary interest was whether subjects would show a reduced orientation effect for rotated drawings that previously had been presented upright but imagined at the orientations at which the drawings were later named. As is evident in Figure 1, the magnitude of the orientation effect did not differ for old and new drawings in the imagined group $(F<1)$. Similarly, the slopes of the linear component of the orientation effect did not 
differ $(F<1)$. The overall effect of orientation was significant $\left[F(5,115)=13.33, M S_{\mathrm{e}}=12,622, p<.001\right]$, and this effect had a strong linear component $[F(1,23)=$ $\left.106.52, M S_{\mathrm{e}}=6,312, p<.001\right\}$. As in the other groups, old drawings were named faster than new drawings $(1,072$ vs. $1,157 \mathrm{msec})\left[F(1,23)=14.53, M S_{\mathrm{e}}=35,288, p<\right.$ $.001]$. The corresponding percent error rates for the six orientations were $8.9 \%, 7.3 \%, 9.4 \%, 8.6 \%, 7.0 \%$, and $7.4 \%$ for old drawings, and for new, $9.0 \%, 8.9 \%, 10.0 \%$, $8.8 \%, 8.5 \%$, and $9.3 \%$.

An analysis comparing the upright and imagined groups showed that the orientation effect did not vary as a function of group or item type $(F<1)$. The two groups did differ in the size of the advantage seen for old over new drawings $\left[F(1,46)=39.97, M S_{\mathrm{e}}=23,572, p<.001\right]$. For the upright group, this advantage was $246 \mathrm{msec}$, whereas for the imagined group, the advantage was only $85 \mathrm{msec}$.

The time to form an image in the training phase for the imagined group is shown in Figure 2. Image formation time was analyzed in a repeated measures ANOVA with block and orientation as factors. In this analysis, the data for $180^{\circ}$ were included in the assessment of the linear component of the orientation effect, since there was no a priori reason for their exclusion. Additional post hoc analyses considering only the data between $0^{\circ}$ and $\pm 120^{\circ}$ yielded identical results to those reported below.

The analysis with contrast weights of $-3,-1,1,3$, $1,-1$ applied to the means for the six orientations revealed a significant effect of orientation $[F(5,115)=$ $\left.26.56, M S_{\mathrm{e}}=734,138, p<.001\right]$, which had a strong linear component $\left[F(1,23)=28.12, M S_{\mathrm{e}}=1,007,481, p<\right.$ $.001]$. As is evident, there was a significant deviation from linearity $\left[F(4,92)=25.97, M S_{\mathrm{e}}=665,802, p<\right.$ $.001]$, most clearly evidenced at $180^{\circ}$. Overall performance improved over blocks $\left[F(4,92)=28.44, M S_{\mathrm{e}}=\right.$ $1,670,502, p<.001]$, and the orientation effect also changed across blocks $\left[F(20,460)=3.71, M S_{\mathrm{e}}=\right.$ $165,054, p<.001]$. The magnitude of the linear effect of orientation was reduced with practice $[F(4,92)=3.18$, $\left.M S_{\mathrm{e}}=156,214, p<.017\right]$. But even in the final block, the orientation effect was robust $[F(5,115)=10.54$, $\left.M S_{\mathrm{e}}=125,434, p<.001\right]$, with a strong linear component $\left[F(1,23)=12.12, M S_{\mathrm{e}}=166,882, p<.002\right]$, and displayed the characteristic M-shaped function often found for identification of drawings named for the first time.

\section{DISCUSSION}

There are two main findings. First, rotating images of upright objects to nonupright orientations does not result in the formation of long-term representations that can be used for orientation-invariant identification of the same objects when they are finally presented rotated. When objects were named at orientations at which they previously had been imagined, no effects of the prior experience were found; equivalent and robust effects of orientation on naming time were found for both previously imagined and new objects. Second, the function relating image formation time to orientation is $\mathbf{M}$-shaped and resembles that obtained for naming of rotated objects.

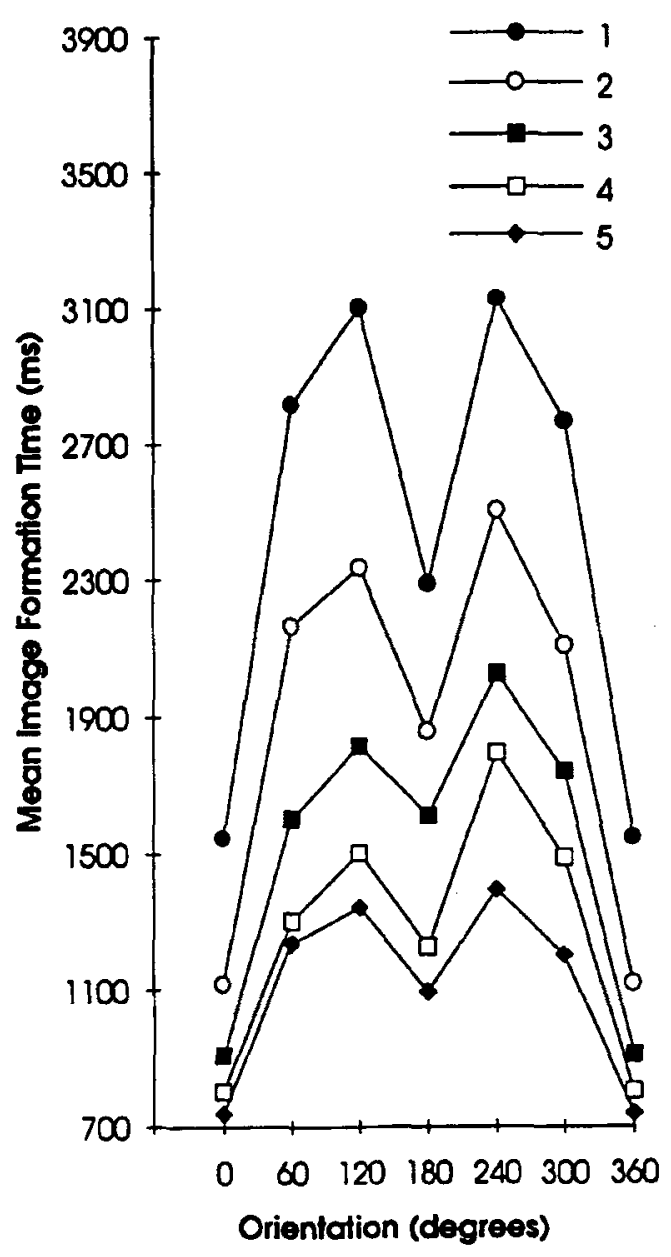

Figure 2. Mean image formation time in each block at each orientation for the imagined group in the training phase.

The accumulating evidence (Jolicoeur \& Milliken, 1989; Murray et al., 1993) seems to favor the view that, in general, reduced orientation effects are the result of the use of orientation-invariant information. Through repeated experience with the objects in the experimental set, individuals learn to isolate orientation-invariant parts or attributes which reliably permit discrimination among the objects for relatively orientation-free identification. However, the earlier work was unable to rule out entirely the multiple-representations explanation of the practice effect for natural objects previously viewed upright in the context of rotated objects (Jolicoeur \& Milliken, 1989) or for rotated objects in general (Murray et al., 1993), if forming multiple imagined representations presented a viable identification strategy. The present study demonstrates that this is not a likely strategy and argues against an account based on multiple orientation-specific representations formed following the imagining of rotated objects.

In the present study, subjects in the imagined condition did not name the upright objects when they imagined them at nonupright orientations. This is in contrast to the rotated condition, in which subjects named the objects in the training phase. This raises the possibility that the observed lack of an attenuation of the orientation effect in the subsequent identification task for the imagined group was due to the absence of a naming response in the earlier imagining task. It is possible that the naming response itself is a necessary part of the representation that is formed following experience with the objects, and that without this naming response, formation of the appropriate representations that result in the attenuation of the orientation effect does not occur. 
Two sets of data bear on this issue. In one study, subjects made leftright judgments of rotated drawings of objects over repeated blocks of trials (Jolicoeur, 1985), whereas in another, top-bottom discriminations were performed (McMullen \& Jolicoeur, 1992). In neither study were subjects additionally asked to name the objects presented repeatedly in the context of the discrimination tasks. However, in a final, surprise block of trials, subjects were asked to name the objects experienced in the earlier discrimination tasks, and the magnitude of the effect of orientation on naming was significantly reduced. These data indicate that producing a naming response during early experience of the objects is not a prerequisite for observing an attenuated orientation effect when the objects are subsequently named.

It appears that the processes giving rise to the attenuation of the orientation effect on naming are enacted in identification, left-right discrimination, and top-bottom discrimination tasks. One process may be the identification of orientation-invariant information. The development of sensitivity to orientation-invariant information with practice, however, does not occur as a default strategy, insofar as it only occurs for upright objects when they are encoded in the context of rotated objects (Jolicoeur \& Milliken, 1989). The present results suggest further that the processes involved in the formation of images at nonupright orientations is also not sufficient to activate a strategy of associating different parts or attributes with particular objects.

The time to imagine an object rotated to a particular orientation was proportional to the difference between the upright object and the orientation of the required image. This suggests that subjects did mentally rotate the upright object to form an image, as has been found in other studies using the image preparation procedure (e.g., Bethell-Fox \& Shepard, 1988; Cooper \& Shepard, 1973). However, a notable feature of the image formation function was its " $M$ " shape, with RTs at $180^{\circ}$ being much faster than would be expected on the basis of RTs for other orientations. This represents a deviation from the mental rotation functions observed for left-right decisions, but it is very similar to the function typically observed for naming rotated objects.

What might account for the faster RTs at $180^{\circ}$ for naming and image formation tasks? An answer, perhaps, lies in consideration of how an inverted image might be brought to the upright or vice versa. One possibility is that subjects may rotate the objects through the depth plane, where rotation occurs about the horizontal axis (this could also be considered a reflection about the horizontal axis). Bressan and Vicario (1984) have reported a preference on the part of subjects for rotation in the depth plane rather than rotation in the image plane for $180^{\circ}$ stimuli. Preliminary work in my laboratory has indicated that depth plane rotation is over $200 \mathrm{msec}$ faster than image plane rotation. This suggests that in naming tasks, and in the image formation task of the present study, subjects are engaging in rotation through the depth plane and not the image plane. This possibility of rotation through the depth plane for identification of inverted objects appears worthy of further study.

\section{REFERENCES}

Bethell-Fox, C. E., \& ShePard, R. N. (1988). Mental rotation: Effects of stimulus complexity and familiarity. Journal of Experimental Psychology: Human Perception and Performance, 14, 12-23.

Bressan, P., \& Vicario, G. B. (1984). On misoriented letters. Perception, 13, 687-694.

COOPER, L. A. (1975). Mental rotation of random two-dimensional shapes. Cognitive Psychology, 3, 20-43.

COOPER, L. A., \& SHEPARD, R. N. (1973). Chronometric studies of the rotation of mental images. In W. G. Chase (Ed.), Visual information processing (pp. 75-176). New I .rk: Academic Press

JolicoeUR, P. (1985). The time to name disoriented natural objects. Memory \& Cognition, 13, 289-303.

JoLICOEUR, P. (1988). Mental rotation and the identification of disoriented objects. Canadian Journal of Psychology, 42, 461-478.

Jolicoeur, P., \& Milliken, B. (1989). Identification of disoriented objects: Effects of context of prior presentation. Journal of Experimental Psychology: Learning, Memory, \& Cognition, 15, 200-210.

MAKI, R. H. (1986). Naming and location of tops of rotated pictures. Canadian Journal of Psychology, 40, 368-387.

MCMullen, P. A., \& Jolicoeur, P. (1992). The reference frame and effects of orientation on finding the top of rotated objects. Journal of Experimental Psychology: Human Perception \& Performance, 18, 807-820.

MURRAY, J. E. (1995). The role of attention in the shift from orientationdependent to orientation-invariant identification of disoriented objects. Memory \& Cognition, 23, 49-58.

Murray, J. E., Jolicoeur, P., McMullen, P. A., \& Ingleton, M. (1993). Orientation-invariant transfer of training in the identification of rotated natural objects. Memory \& Cognition, 21, 604-610.

SCHNEIDER, W. (1988). Micro Experimental Laboratory: An integrated system for IBM PC compatibles. Behavior Research Methods, Instruments, \& Computers, 20, 206-217.

SNODGRASS, J. G., \& VANDERWART, M. (1980). A standardized set of 260 pictures: Norms for name agreement, image agreement, familiarity, and visual complexity. Journal of Experimental Psychology: Human Learning \& Memory, 6, 174-215.

TARR, M. J., \& PinKer, S. (1989). Mental rotation and orientationdependence in shape recognition. Cognitive Psychology, 32, 193254.

(Manuscript received July 25, 1994; revision accepted for publication October 26,1994 .) 